\title{
Mild cerebellar injury does not significantly affect cerebral white matter microstructural organization and neurodevelopmental outcome in a contemporary cohort of preterm infants
}

Richelle E M Senden ${ }^{1}$, Kristin Keunen ${ }^{1,2}$, Niek E van der Aa $a^{1,2}$, Alexander Leemans ${ }^{2,3}$, Ivana Isgum ${ }^{2,3}$, Max A Viergever ${ }^{2,3}$, Jeroen Dudink ${ }^{1,2}$, Linda S de Vries ${ }^{1,2}$, Floris Groenendaal ${ }^{1,2}$ and Manon J N L Benders ${ }^{1,2}$

\begin{abstract}
BACKGROUND: Preterm birth is associated with an increased risk of cerebellar injury. The aim of this study was to assess the impact of cerebellar hemorrhages $(\mathrm{CBH})$ on cerebral white matter microstructural tissue organization and cerebellar volume at term-equivalent age (TEA) in extremely preterm infants. Furthermore, we aimed to evaluate the association between $\mathrm{CBH}$ and neurodevelopmental outcome in late infancy.
\end{abstract}

METHODS: A total of 24 preterm infants with punctate $\mathrm{CBH}$ were included and each matched to two preterm control infants. T1-, T2-weighted images and diffusion-weighted imaging were acquired on a $3 T$ magnetic resonance imaging (MRI) system. Regions of interest were drawn on a populationspecific neonatal template and automatically registered to individual fractional anisotropy (FA) maps. Brain volumes were automatically computed. Neurodevelopmental outcome was assessed using the Bayley scales of Infant and Toddler Development at 2 years of corrected age.

RESULTS: CBHs were not significantly related to FA in the posterior limb of the internal capsule and corpus callosum or to cerebellar volume. Infants with $\mathrm{CBH}$ did not have poorer neurodevelopmental outcome compared with control infants. CONCLUSION: These findings suggest that the impact of mild $\mathrm{CBH}$ on early macroscale brain development may be limited. Future studies are needed to assess the effects of $\mathrm{CBH}$ on long-term neurodevelopment.

$\mathbf{T}$ he impact of neonatal cerebellar injury on cerebellar development is increasingly recognized (1). Preterm infants have been noted to be of particular risk because essential cerebellar maturation takes place under high-risk circumstances instead of the protective environment of the maternal womb (2). The overall incidence of cerebellar injury in preterm infants is now estimated to be $15-20 \%$. Punctate lesions are more frequently detected because of the increasing use of magnetic resonance imaging (MRI) in the preterm population (3).

Literature findings on the effects of cerebellar injury on neurodevelopmental outcome in preterm-born children vary with the severity of cerebellar lesions. Large hemorrhages have been associated with high early mortality rates and severe developmental delay (4-7), whereas the effects of punctate lesions on outcome remain unclear. Tam et al. compared a small group of preterm infants with punctate cerebellar lesions $(n=9)$ with preterm control infants $(n=94)$, and observed a fivefold increased odds ratio of neurologic abnormalities at 3-6 years of age but no differences in cognitive functioning in early childhood (8). In another study, $57 \%$ of preterm-born children with small cerebellar hemorrhages (CBHs) showed abnormal neurodevelopmental outcome at the age of 2 years compared with $48 \%$ of preterm control children (3).

Neurodevelopmental deficits following cerebellar injury in the preterm period are thought to originate from cerebellocerebral diaschisis, which describes loss of function in the supratentorial brain due to damage to the cerebellum (9). In the presence of diaschisis, less cerebral connections may be formed and maintained, leading to diminished neural connectivity $(2,10)$. Such putative structural alterations in the neonatal brain are supported by studies reporting volume reductions of the (contralateral) supratentorial brain in preterm-born children with cerebellar injury or malformations (11-13).

White matter connections can be investigated in detail using diffusion-weighted imaging (DWI), an MRI technique that offers the opportunity to shed light on the impact of early cerebellar injury on white matter microstructural tissue organization (14). The latter may be affected when diaschisis is present and has led to structural alterations. Such secondary alterations in major white matter pathways subsequent to

\footnotetext{
${ }^{1}$ Department of Neonatology, University Medical Center Utrecht and Utrecht University, Utrecht, The Netherlands; ${ }^{2}$ Brain Center Rudolf Magnus, University Medical Center Utrecht and Utrecht University, Utrecht, The Netherlands; ${ }^{3}$ Image Sciences Institute, University Medical Center Utrecht and Utrecht University, Utrecht, The Netherlands. Correspondence: Manon J.N.L. Benders (m.benders@umcutrecht.nl) 


\section{Limited impact small cerebellar injury Articles}

cerebellar injury may ultimately impair cognitive and motor functioning later in life.

The aim of this study was to investigate the association between cerebellar lesions and white matter tissue organization of major white matter structures, including the corpus callosum and bilateral posterior limb of the internal capsule (PLIC) in a cohort of very preterm infants. These structures were selected because they have both been reported to have implications for motor and cognitive functioning (15-17). The PLIC has been reported to show early fractional anisotropy (FA) changes in the developing brain, indicating that this structure rigorously matures during the neonatal period (14), and the corpus callosum is the largest white matter fiber bundle. As such, global white matter changes are likely to be easily detected here. As a secondary objective, the association between cerebellar injury and cerebellar volume was investigated. Thirdly, the association between cerebellar injury and neurodevelopmental outcome at the age of 2 years was investigated. We hypothesized that cerebellar injury would be associated with impaired white matter maturation, reflected by reduced FA at term-equivalent age (TEA), smaller cerebellar volumes, and adverse neurodevelopmental outcome in late infancy.

\section{METHODS}

\section{Patients}

Infants included in this nested case-control study were enrolled in a prospective cohort study performed at the Wilhelmina Children's Hospital, University Medical Center Utrecht between May 2008 and March 2013 in case the infant was born $<28$ weeks of gestation and when at least one MRI was obtained during the neonatal period. Infants with chromosomal abnormalities, congenital anomalies, or infections of the central nervous system were excluded. Subjects were eligible for inclusion in the present study in case cerebellar injury was present and when good-quality DWI data were available at TEA $(n=24)$. Each infant was matched to two control infants without cerebellar injury $(n=48)$. Matching criteria were the following: sex, gestational age (GA; weeks), small for GA (birth weight $z$-score below -1.28), grade of intraventricular hemorrhage (IVH) assessed using early and serial cranial ultrasound and MRI, and year of birth. All neuroimaging data were obtained as part of a clinical protocol and no infants were exposed to research procedures. Therefore, written informed parental consent for use of the clinically acquired data was waived by the Institutional Review Board of the University Medical Center Utrecht, the Netherlands. The Institutional Review Board approved use of the clinically acquired data for purposes of this study. A flowchart of the selection procedure is provided in Supplementary Figure S1 online.

\section{Magnetic Resonance Imaging}

All MRI investigations were performed on a 3-Tesla MR system at TEA (Achieva, Philips Healthcare Medical Systems, Best, the Netherlands), using an eight-channel sense head coil. Sedation was administered before scanning (oral chloral hydrate $50-60 \mathrm{mg} / \mathrm{kg}$ ). Infants received earmuffs for hearing protection (Natus Medical, San Carlos, CA) and were placed in a vacuum fixation pillow to reduce head movement (Kohlbrat and Bunz, Radstadt, Austria). A neonatologist or physician assistant was present throughout the entire examination and continuously monitored heart rate, respiration rate, and transcutaneous oxygen saturation.

The MRI protocol included coronal three-dimensional T1weighted (repetition time (TR) $9.5 \mathrm{~ms}$, echo time (TE) $4.6 \mathrm{~ms}$, voxel size $1.2 \times 0.78 \times 0.91 \mathrm{~mm})$ and coronal T2-weighted images (TR 4,847 ms, TE $150 \mathrm{~ms}$, voxel size $1.2 \times 0.35 \times 0.35 \mathrm{~mm}$ ). The DWI protocol included axial single-shot spin-echo echoplanar imaging (SENSE P-reduction 2, TR $7,745 \mathrm{~ms}$, TE $48 \mathrm{~ms}$, voxel size $1.41 \times 1.41 \times 2 \mathrm{~mm}^{3}$, and total scan time $4.28 \mathrm{~min}$ ). DWI images were obtained in 32-noncollinear directions with a $b$-value of $800 \mathrm{~s} /$ $\mathrm{mm}^{2}$ and one non-diffusion-weighted image. CBHs were assessed using the cerebellar injury score proposed by Kidokoro et al. (18) on T2- and T1-weighted images and were classified as punctate unilateral injury ( $<6$ punctate lesions), punctate bilateral injury ( $\geq 6$ punctate lesions), unilateral extensive or bilateral extensive bilateral, and/or with vermis involvement. White matter injury was assessed according to Woodward et al. (19) and was classified as no injury, mild, moderate, or severe abnormalities. The Woodward score system assesses signal abnormality, cystic abnormalities, ventricular dilatation, volume loss of the periventricular white cerebral matter, and thinning of the corpus callosum. Two neonatologists (L.d.V. and M.B.) experienced in neonatal neuroimaging evaluated all scans with respect to neonatal brain injury.

\section{Image Processing}

DWI data were analyzed using ExploreDTI (http://exploredti.com) (20). Processing included registration of the DWI to the $b=0$ image, followed by correction for subtle subject motion and eddy currentinduced geometric distortions (21). The diffusion tensor was fitted using a robust fitting model (22). All diffusion MRI images were visually inspected to ensure good data quality. Data sets were included when five or less DWI volumes had motion artifacts. Next, regions of interest were placed on a population-specific neonatal atlas that was created using the Diffusion Tensor Imaging ToolKit (http:// dti-tk.sourceforge.net). The Diffusion Tensor Imaging ToolKit allows tensor-based registration using a rigid, affine, and finally a deformable alignment. The population-specific atlas was created by averaging FA maps of 10 preterm control infants born at the Wilhelmina Children's Hospital. These 10 infants did not show any apparent brain injury on their TEA MRI and had no or mild white matter abnormalities according to the scoring system by Woodward et al. (19). Regions of interest were manually placed on the population-specific neonatal template in the corpus callosum, and the left and right PLIC using the Freesurfer Virtualbox (http://surfer. nmr.mgh.harvard.edu) (23) (Figure 1), and were registered to each individual data set using ExploreDTI (20). The entire corpus callosum was delineated as one region of interest (i.e., including the genu, body, and splenium). Atlas registration was visually inspected for all subjects and FA values were automatically derived by following the registration procedure.

\section{Brain Volumes}

Brain tissue segmentation was automatically performed using an inhome developed and previously published automated segmentation
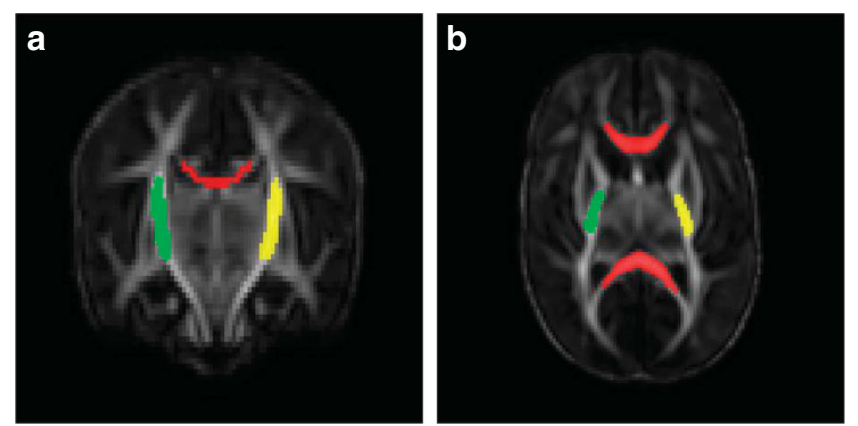

Figure 1. Region of interest placement on FA-map. The corpus callosum (red) and PLIC (right PLIC in green, left PLIC in yellow) were manually delineated on the group-specific neonatal atlas (see Methods section for further details). Figure 1 illustrates regions of interest in the coronal (a) and axial (b) planes. PLIC, posterior limb of the internal capsule. 


\section{Articles | Senden et al.}

method (24). This method segments eight different tissue classes, of which cerebellar volume and intracranial volume were selected for the purpose of this study. Good-quality cerebellar volumes were available for 20 infants with cerebellar injury (83\%) and 41 control infants (85\%).
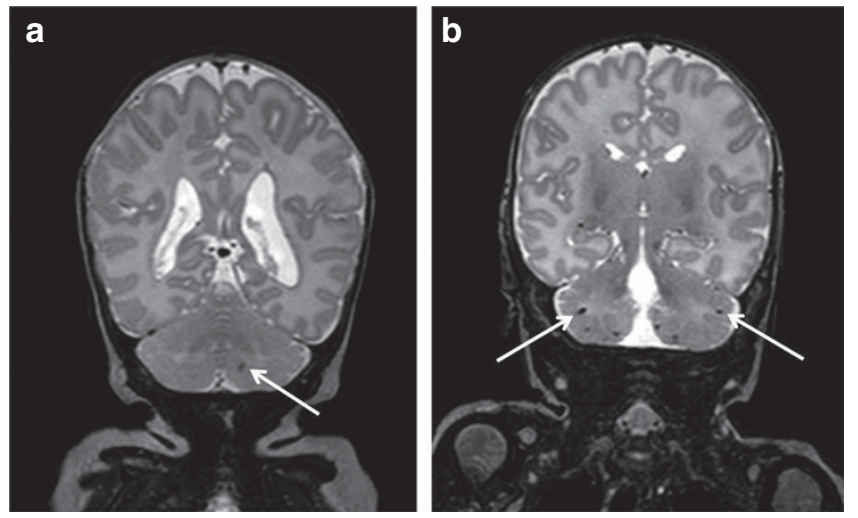

Figure 2. Cerebellar lesions on coronal T2-weighted image. (a) Illustration of unilateral punctate lesions $(n=16)$. (b) Depicts bilateral punctate lesions $(n=8)$. MRI, magnetic resonance imaging.

\section{Neurodevelopmental Assessment}

Neurodevelopmental outcome was assessed at the outpatient clinic of the Wilhelmina Children's Hospital. At 2 years of corrected age, cognition and motor function were assessed by a developmental specialist using the Bayley scales of Infant and Toddler Development, Third Edition (BSITD-III; $n=65,90 \%$ ) (25). As the attention span is generally short at this age, only cognitive composite scores and fine and gross motor scores were obtained to acquire reliable results. All scores were corrected for prematurity. Normative means for cognitive scores were 100 with a SD of 15 . For motor function normative means were 10 with a SD of 3 .

\section{Data Analysis}

Data analysis was performed using IBM SPSS Statistics version 22.0 (SPSS, Chicago, IL). Clinical variables of cases and controls were compared using one-way repeated measures ANOVA for parametric data and using the Friedman test for non-parametric data. The association between cerebellar injury and FA values measured at TEA, cerebellar volume (corrected for intracranial volume) measured at TEA, and neurodevelopmental outcome at 2 years of age (BSITDIII) was evaluated using one-way repeated measures ANOVA with a Greenhouse-Geisser correction in order to compare the case infant with its two matched control infants. In case results reached statistical significance, with an alpha level set to $<0.05$, Bonferroni correction was applied to identify significant groups. Maternal

Table 1. Clinical characteristics

\begin{tabular}{|c|c|c|c|}
\hline & Infants with cerebellar hemorrhage $(n=24)$ & Control infants $(n=48)$ & $P$ value \\
\hline Sex (male, n (\%)) & $13(54 \%)$ & $26(54 \%)$ & \\
\hline Gestational age (weeks, mean (range)) & $26.3(24.3-27.9)$ & $26.3(24.1-28.0)$ & \\
\hline Birth weight $z$-score (mean (range)) & $0.2(-1.5-1.7)$ & $0.2(-2.4-2.0)$ & \\
\hline Mechanical ventilation (days, mean (range)) & $13(0-41)$ & $11(0-42)$ & 0.40 \\
\hline Necrotizing enterocolitis requiring surgery (n (\%)) & $2(8 \%)$ & $0(0 \%)$ & 0.16 \\
\hline Culture-proven sepsis (n (\%)) & $15(63 \%)$ & $17(35 \%)$ & 0.12 \\
\hline \multicolumn{4}{|l|}{ Intraventricular hemorrhage (n (\%)) } \\
\hline Grade IV & $3(13 \%)$ & $3(6 \%)$ & \\
\hline White matter injury (n (\%)) & & & 0.88 \\
\hline No or mild abnormalities & $19(79 \%)$ & $39(81 \%)$ & \\
\hline Moderate or severe abnormalities & $5(21 \%)$ & $9(19 \%)$ & \\
\hline Post-hemorrhagic ventricular dilatation requiring intervention (n (\%)) & $3(13 \%)$ & $5(10 \%)$ & 0.25 \\
\hline Postmenstrual age at scan (weeks, mean (range)) & $42.2(40.3-43.4)$ & $41.1(39.3-42.7)$ & 0.36 \\
\hline Maternal education $(n(\%))^{\mathrm{a}}$ & & & 0.81 \\
\hline
\end{tabular}

${ }^{a}$ Maternal education was classified according to the Dutch Central Bureau of Statistics classification. 
education level and related socioeconomic status are well known to have an impact on cognitive functioning, especially in infancy (26). Therefore, differences in maternal education level were tested between infants with $\mathrm{CBHs}$ and the control group using the Friedman test. There were no significant differences in level of maternal education between the groups, and therefore this factor was not included in the models evaluating neurodevelopmental outcome. A $P$ value $<0.05$ was considered statistically significant for all analyses.

\section{RESULTS}

A total of 24 preterm infants with punctate CBHs (unilateral $n=16$, bilateral $n=8$ ) were eligible for inclusion as shown in Figure 2. None of the infants had cerebellar vermis involvement. Matching was successfully performed, as there were no significant differences in sex, GA, birth weight, and IVH between the two groups. In addition, there were no significant differences in clinical characteristics between infants with $\mathrm{CBH}$ s and control infants (Table 1). Cultureproven sepsis showed a trend toward being more prevalent in infants with $\mathrm{CBHs}$, but the difference was not statistically significant ( $63 \%$ vs. $35 \%, P=0.12$ ). One control infant died after the neonatal period because of severe bronchopulmonary dysplasia.

\section{Cerebellar Injury and White Matter Microstructure}

The presence of cerebellar injury was not associated with lower FA values in the corpus callosum or left and right PLIC (FA corpus callosum: case infants mean FA $0.35 \pm 0.04$ (confidence interval (CI) $0.34-0.37$ ) vs. control infants $0.35 \pm 0.04(0.34-0.36), P=0.52, F=0.64$; left PLIC: case infants $0.46 \pm 0.03(0.44-0.47)$ vs. control infants $0.46 \pm 0.03$ (0.45-0.47), $P=0.51, \quad F=0.67$; right PLIC: case infants $0.46 \pm 0.02(0.46-0.47)$ vs. control infants $0.46 \pm 0.04(0.44-$ $0.47), P=0.11, F=2.48$; Figure 3a).

\section{Cerebellar Injury and Volume}

Cerebellar injury was not associated with smaller cerebellar volumes at TEA (cerebellar volume corrected for intracranial volume: case infants mean $5.6 \pm 0.5 \%$ (CI 5.3-5.9) vs. control infants $5.6 \pm 0.5 \%(5.4-5.7), P=0.45, F=0.82$; Figure $3 a)$. Cerebellar injury was not associated with smaller intracranial volume (case infants' mean $502 \pm 50 \mathrm{~cm}^{3}$ (479-526) vs. control infants' mean $482 \pm 63 \mathrm{~cm}^{3}(462-502), P=0.14$, $F=2.13)$.

\section{Neurodevelopmental Outcome}

Cerebellar injury was not associated with poorer cognitive functioning and lower fine and gross motor scores at 2 years of age as shown in Figure $\mathbf{3 b}$ (cognition composite score: case infants' mean $101 \pm 11$ (CI 96-106) vs. control infants $107 \pm 14(102-11), F=2.44, P=0.11$; fine motor score: case infants $12.9 \pm 2.5(11.8-14.0)$ vs. control infants $13.4 \pm 1.8$ (12.8-13.9), $F=0.50, \quad P=0.59$; gross motor score: case infants $9.1 \pm 2.4(8.0-10.2)$ vs. control infants $9.4 \pm 2.4$ (8.610.1), $\quad F=0.73, \quad P=0.25)$. Neurodevelopmental outcome comparisons were not significantly different after regressing
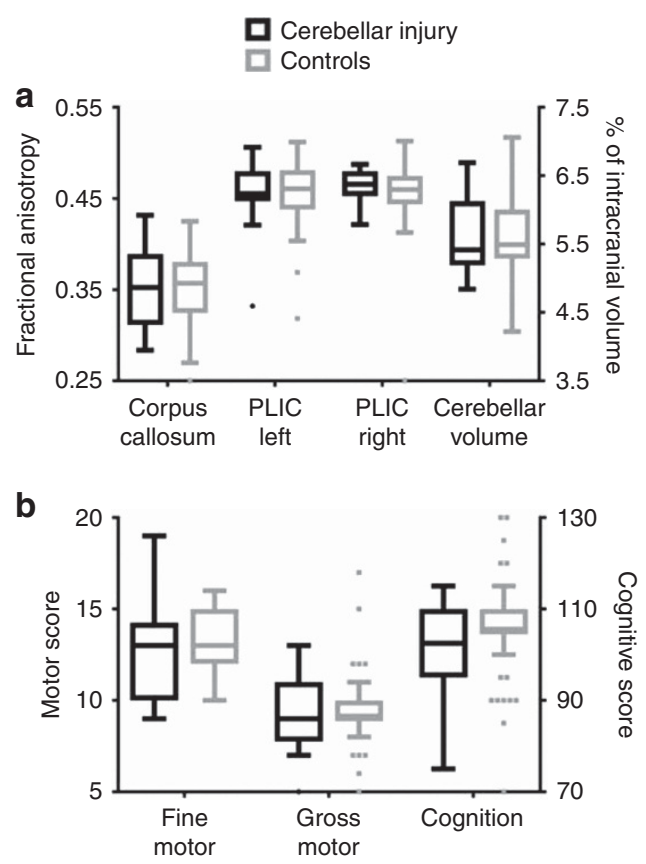

Figure 3. White matter integrity and cerebellar volume and neurodevelopmental outcome. (a) Fractional anisotropy in the corpus callosum (outer left) and PLIC (middle left and middle right) in infants with cerebellar injury (black) and control infants (gray). Cerebellar volume at term-equivalent age in infants with cerebellar injury (black) and control infants (gray) is shown in the outer right. (b) The results of the BSITD-III (motor scaled scores outer left and middle; cognition outer right) as assessed at the age of 2 years ( $n=65$ children)(25). There were no significant differences in BSITD-III scores between children with cerebellar hemorrhages (black) and matched controls without cerebellar hemorrhages (gray). BSITD-III, Bayley scales of Infant and Toddler Development, Third Edition; PLIC, posterior limb of the internal capsule.

out the effect of maternal education (cognition $F=3.56$, $P=0.06$; fine motor $F=0.86, P=0.36$; gross motor $F=0.24$, $P=0.63)$. Finally, we compared infants with bilateral lesions, unilateral lesions, and no lesions, and found no statistically significant differences in clinical characteristics or any of the included outcome parameters.

\section{DISCUSSION}

In the present case-control study, the impact of punctate cerebellar lesions on white matter tissue organization, cerebellar volume, and neurodevelopmental outcome was investigated in very preterm infants. Cerebellar lesions were not related to FA in the bilateral PLIC and corpus callosum at TEA, revealing no significant impact of preterm cerebellar injury on white matter integrity of these structures. Moreover, cerebellar injury was not associated with cerebellar volume at TEA. As a third objective, the association between cerebellar lesions and neurodevelopmental outcome was evaluated. Cerebellar lesions were not related to cognitive, fine, and gross motor functioning at 2 years of age. Our findings suggest that the effects of mild cerebellar lesions on supratentorial white matter development and subsequent cognitive and overall motor functioning in late infancy may be limited. 


\section{Articles | Senden et al.}

We did not observe structural cerebral white matter loss in the presence of cerebellar injury. A number of explanations could account for these differences. First, cerebellar injury consisted of punctate lesions and remote effects may have been too small to affect the structural maturation of the corpus callosum and corticospinal tracts. Second, the time period between the occurrence of cerebellar injury and the MRI at TEA may have been too short. It remains elusive whether remote effects may become apparent beyond TEA.

Studies on posterior fossa tumors in children have linked cerebellar injury to neurological symptoms of cerebral dysfunctioning, putting forward cerebellocerebral diaschisis as the underlying phenomenon (27-29). In these studies children developed mutism and apraxia of the oral pharyngeal musculature a few days after the resection of a tumor affecting the cerebellum $(27,28)$. Notably, reduced cerebral blood flow in the thalami, frontal, and temporal lobes was also observed in a child who developed mutism after cerebellar medulloblastoma resection and may thus have contributed to the neurological signs and symptoms, rather than cerebellocerebral diaschisis (29).

In preterm infants, literature on the potential impact of cerebellar injury on cerebral structures is limited. One study reported decreased contralateral cerebral brain volume at TEA in 10 preterm infants with unilateral isolated cerebellar injury (11). Contrary to our study, all infants had large cerebellar lesions as they were diagnosed by means of cranial ultrasound. Detection of small cerebellar lesions is notoriously difficult using cranial ultrasound (8,30). As extensive cerebellar injury has been noted to have an impact on cerebral brain growth, large CBHs may also have a negative effect on white matter maturation in the corpus callosum and PLIC, whereas small lesions may not. Alternatively, decreased cerebral brain volume in the presence of large CBHs may have been the result of direct effects of blood products on the supratentorial brain rather than of remote effects from reduced input into the cerebrum (2). Evidence for this alternative hypothesis comes from preterm infants with IVH. In the presence of IVH, cerebellar underdevelopment observed with MRI has been related to hemosiderin deposition (31). Similarly, hemosiderin might lead to decreased cerebral brain volume in the presence of large $\mathrm{CBHs}$ as well. In the present cohort of preterm infants with small CBHs, we did not observe smaller cerebellar volumes. Given the notion that detrimental effects on growth have only been reported in the presence of large hemorrhages (11), the lesions as observed in our study may have been too small to exert such effects. The notion that cerebellar injury did not directly affect cerebellar volume may also support the finding that cerebellar injury was not associated with white matter tissue organization in our study: in the absence of direct detrimental effects of $\mathrm{CBHs}$, remote cerebral effects are likely limited.

The current findings did not provide evidence for a significant impact of cerebellar injury on neurodevelopmental outcome at 2 years of age in children with mild cerebellar lesions. Our findings are in line with literature reporting on punctate cerebellar lesions. In a prospective study in 108 preterm infants with $(n=16)$ and without cerebellar injury $(n=92)$, small CBHs, as detected using MRI at TEA, were not associated with adverse neurodevelopmental outcome at 2 years of age (3). Conversely, large cerebellar lesions (as diagnosed by cranial ultrasound) have been related to deficits in cognitive functioning and motor performance in pretermborn children. In addition, children with large CBHs showed behavioral problems and early signs of autism at 3233 months of age in two studies by Limperopoulos et al. $(7,10)$. These social functions are governed by higher-order cortical regions, which are modulated by the cerebellum (32-34). Disruption of cerebellocerebral connections by large hemorrhages may lead to impaired modulation of signaling from higher-order association cortices, which may lead to symptoms of deficits in higher cognitive functions that do not become apparent until well into school age (35). Similarly, small cerebellar lesions may interfere with higher-order cognitive functioning, although their effects are likely less disruptive. Rigorous follow-up through childhood and adolescence is needed to evaluate the full impact of $\mathrm{CBHs}-$ including small lesions-on cognitive and behavioral functioning. Here, we demonstrate that small CBHs do not affect neurodevelopment in infancy, as there were no differences in outcome scores on the BSITD-III at age of 2 years.

Some strengths and limitations need to be taken into consideration when interpreting the results of the present study. We were able to match case and control infants by sex, GA, birth weight, year of birth, and IVH. IVH was previously reported to reduce FA values of the corpus callosum in very preterm infants, and may therefore confound the primary outcome parameter in case matching by this criterion would be omitted (15). Furthermore, loss-to-follow-up was limited as $90 \%$ of infants returned for their follow-up visit at the age of 2 .

The sample size was small, because of the limited incidence of CBHs in our NICU population despite having collected data over a 5-year period. A multicenter study could potentially increase the power of future studies to detect significant effects; however, at present limited interscanner comparability of DWI parameters hinders this solution (36). Consequently, results of our study may reflect a lack of statistical power rather than a true lack of effect (37). However, it is difficult to calculate the appropriate sample size because most studies investigating the impact of cerebellar injury on neurodevelopmental outcome in preterm infants include large cerebellar lesions (5-7). Future studies should thus have a prospective design in a multicenter setting with specific attention to the uniformity of scanning protocols and MRI scanners. A second limitation is that the clinical DWI protocol employed here is not able to accurately reconstruct fiber bundles in voxels where multiple fibers cross. DWI techniques such as high-angular-resolution diffusion imaging may provide more detailed insight into the effect of cerebellar injury on the cerebral white matter. These DWI techniques 
allow reliable reconstruction of complex fiber orientations. It follows that, when using these techniques, cerebellocerebral tracts may be evaluated in detail and potential effects on gray matter organization may be studied as well $(38,39)$. In addition, the effect of cerebellar injury on cerebellar microstructure may be investigated. Future research should focus on advanced DWI techniques, may consider additional post-processing DWI procedures, including tract-based spatial statistics, and extend the follow-up period through childhood and adolescence (40).

\section{CONCLUSION}

The present study suggests that the impact of mild cerebellar lesions on supratentorial white matter microstructural organization, cerebellar volume, and neurodevelopmental outcome in late infancy may be limited. Further research is warranted to corroborate these findings in a larger population of preterm infants with cerebellar lesions, to evaluate the impact of severe cerebellar injury on the developing white matter and cerebellum, and to gain insight into the potential long-term consequences of cerebellar injury on higher cognitive functioning. Such studies should ideally be of large sample sizes. We therefore stress the importance of multicenter studies in this context.

\section{SUPPLEMENTARY MATERIAL}

Supplementary material is linked to the online version of the paper at http://www.nature.com/pr

\section{ACKNOWLEDGMENTS}

We thank Nathalie H.P. Claessens and Karina J. Kersbergen for their contribution toward the data collection. We would also like to thank Ingrid C. van Haastert, for her careful evaluation at the outpatient clinic of the children included in this study.

\section{STATEMENT OF FINANCIAL SUPPORT}

R.S. was awarded a Van Walree Scholarship of the Royal Academy of Arts and Sciences (Koninklijke Nederlandse Academie van Wetenschappen) to present results of this study at the Pediatric Academic Societies Meeting in San Diego in 2015. K.K. is supported by a grant from the Wilhelmina Children's Hospital Research Fund (Vrienden WKZ) to Martijn P. van den Heuvel. The research of A.L. is supported by VIDI Grant 639.072.411 from the Netherlands Organization for Scientific Research (NWO).

Disclosure: The authors declare no competing financial interests.

\section{REFERENCES}

1. Brossard-Racine M, du Plessis AJ, Limperopoulos C. Developmental cerebellar cognitive affective syndrome in ex-preterm survivors following cerebellar injury. Cerebellum 2015;14:151-64.

2. Volpe JJ. Cerebellum of the premature infant: rapidly developing, vulnerable, clinically important. J Child Neurol 2009;24:1085-4.

3. Steggerda SJ, De Bruine FT, van den Berg-Huysmans AA, et al. Small cerebellar hemorrhage in preterm infants: perinatal and postnatal factors and outcome. Cerebellum 2013;12:794-801.

4. Limperopoulos C, Benson CB, Bassan H, et al. Cerebellar hemorrhage in the preterm infant: ultrasonographic findings and risk factors. Pediatrics 2005;116:717-24.

5. Dyet LE, Kennea N, Counsell SJ, et al. Natural history of brain lesions in extremely preterm infants studied with serial magnetic resonance imaging from birth and neurodevelopmental assessment. Pediatrics 2006;118:536-48.
6. Bednarek N, Akhavi A, Pietrement C, Mesmin F, Loron G, Morville P. Outcome of cerebellar injury in very low birth-weight infants: 6 case reports. J Child Neurol 2008;23:906-11.

7. Limperopoulos C, Bassan H, Gauvreau K, et al. Does cerebellar injury in premature infants contribute to the high prevalence of long-term cognitive, learning, and behavioral disability in survivors? Pediatrics 2007;120:584-93.

8. Tam EW, Rosenbluth G, Rogers EE, et al. Cerebellar hemorrhage on magnetic resonance imaging in preterm newborns associated with abnormal neurologic outcome. J Pediatr 2011;158:245-50.

9. Monakow CV, Monakow CV. Brain and behavior I: mood, states and mind. In: Pibram KH, ed. Diaschisis. Penguin Books, Baltimore 1969: 27-36.

10. Limperopoulos C, Chilingaryan G, Sullivan N, Guizard N, Robertson RL, $\mathrm{du}$ Plessis AJ. Injury to the premature cerebellum: outcome is related to remote cortical development. Cereb Cortex 2014;24:728-36.

11. Limperopoulos C, Soul JS, Haidar H, et al. Impaired trophic interactions between the cerebellum and the cerebrum among preterm infants. Pediatrics 2005;116:844-50.

12. Limperopoulos C, Chilingaryan G, Guizard N, Robertson RL, Du Plessis AJ. Cerebellar injury in the premature infant is associated with impaired growth of specific cerebral regions. Pediatr Res 2010;68:145-50.

13. Bolduc ME, Du Plessis AJ, Evans A, et al. Cerebellar malformations alter regional cerebral development. Dev Med Child Neurol 2011;53:1128-34.

14. Huppi PS, Dubois J. Diffusion tensor imaging of brain development. Semin Fetal Neonatal Med 2006;11:489-97.

15. Thompson DK, Inder TE, Faggian N, et al. Corpus callosum alterations in very preterm infants: perinatal correlates and 2 year neurodevelopmental outcomes. Neuroimage 2012;59:3571-81.

16. Rose J, Cahill-Rowley K, Vassar R, et al. Neonatal brain microstructure correlates of neurodevelopment and gait in preterm children 18-22 mo of age: an MRI and DTI study. Pediatr Res 2015;78:700-8.

17. van Kooij BJ, de Vries LS, Ball G, et al. Neonatal tract-based spatial statistics findings and outcome in preterm infants. AJNR Am J Neuroradiol 2012;33:188-94.

18. Kidokoro H, Neil JJ, Inder TE. New MR imaging assessment tool to define brain abnormalities in very preterm infants at term. AJNR Am J Neuroradiol 2013;34:2208-14.

19. Woodward LJ, Anderson PJ, Austin NC, Howard K, Inder TE. Neonatal MRI to predict neurodevelopmental outcomes in preterm infants. N Engl J Med 2006;355:685-94.

20. Leemans A, Jeurissen B, Sijbers J, Jones DK. ExploreDTI: a graphical toolbox for processing, analyzing, and visualizing diffusion MR data. Proceedings of the 17th Scientific Meeting, International Society for Magnetic Resonance in Medicine, Honolulu, USA, 2009, 3537.

21. Leemans A, Jones DK. The B-matrix must be rotated when correcting for subject motion in DTI data. Magn Reson Med 2009;61:1336-49.

22. Tax CM, Otte WM, Viergever MA, Dijkhuizen RM, Leemans A. REKINDLE: robust extraction of kurtosis INDices with linear estimation. Magn Reson Med 2015;73:794-808.

23. Reuter M, Schmansky NJ, Rosas HD, Fischl B 2012 Within-subject template estimation for unbiased longitudinal image analysis. Neuroimage 61:1402-8.

24. Anbeek P, Isgum I, van Kooij BJ, et al. Automatic segmentation of eight tissue classes in neonatal brain MRI. PLoS ONE 2013;8:e81895.

25. Bayley N. Bayley Scales of Infant and Toddler Development-Third Edition. Harcourt Assessment NCS. San Antonio, TX: Pearson, 2006.

26. Ball G, Pazderova L, Chew A, et al. Thalamocortical connectivity predicts cognition in children born preterm. Cereb Cortex 2015;25:4310-8.

27. Pollack IF, Polinko P, Albright AL, Towbin R, Fitz C. Mutism and pseudobulbar symptoms after resection of posterior fossa tumors in children: incidence and pathophysiology. Neurosurgery 1995;37:885-93.

28. Dailey AT, McKhann GM 2nd, Berger MS. The pathophysiology of oral pharyngeal apraxia and mutism following posterior fossa tumor resection in children. J Neurosurg 1995;83:467-75. 


\section{Articles | Senden et $a$ l.}

29. Sagiuchi T, Ishii K, Aoki Y, et al. Bilateral crossed cerebello-cerebral diaschisis and mutism after surgery for cerebellar medulloblastoma. Ann Nucl Med 2001;15:157-60.

30. Steggerda SJ, Leijser LM, Wiggers-de Bruine FT, van der Grond J, Walther FJ, van Wezel-Meijler G. Cerebellar injury in preterm infants: incidence and findings on US and MR images. Radiology 2009;252:190-9.

31. Messerschmidt A, Prayer D, Brugger PC, et al. Preterm birth and disruptive cerebellar development: assessment of perinatal risk factors. Eur J Paediatr Neurol 2008;12:455-60.

32. Castelli F, Frith C, Happe F, Frith U. Autism, Asperger syndrome and brain mechanisms for the attribution of mental states to animated shapes. Brain 2002;125:1839-49.

33. Holdefer RN, Miller LE, Chen LL, Houk JC. Functional connectivity between cerebellum and primary motor cortex in the awake monkey. J Neurophysiol 2000;84:585-90.

34. Schmahmann JD. Cognition and the cerebellum. Neurology 2004;63: 1991.
35. Ochiai M, Ichiyama M, Iwayama M, Sakai Y, Yoshida K, Hara T. Longitudinal study of very low birth weight infants until 9years of age; attention deficit hyperactivity and autistic features are correlated with their cognitive functions. Early Hum Dev 2015;91:783-6.

36. Fushimi Y, Miki Y, Okada T, et al. Fractional anisotropy and mean diffusivity: comparison between $3.0-\mathrm{T}$ and $1.5-\mathrm{T}$ diffusion tensor imaging with parallel imaging using histogram and region of interest analysis. NMR Biomed 2007;20:743-8.

37. Altman DG, Bland JM. Absence of evidence is not evidence of absence. Br Med J 1995;311:485.

38. Pieterman K, Batalle D, Dudink J, et al. Cerebello-cerebral connectivity in the developing brain. Brain Struct Funct 2016;222:1625-34.

39. Xu G, Takahashi E, Folkerth RD, et al. Radial coherence of diffusion tractography in the cerebral white matter of the human fetus: neuroanatomic insights. Cereb Cortex 2014;24:579-92.

40. Smith SM, Jenkinson M, Johansen-Berg H, et al. Tract-based spatial statistics: voxelwise analysis of multi-subject diffusion data. Neuroimage 2006;31:1487-505. 